\title{
Wideband, Multiband, Tunable, and Smart Antenna Systems for Mobile and UWB Wireless Applications 2014
}

\author{
Renato Cicchetti, ${ }^{1}$ Antonio Faraone, ${ }^{2}$ Diego Caratelli, ${ }^{3}$ and Massimiliano Simeoni ${ }^{4}$ \\ ${ }^{1}$ Department of Information, Electronic and Telecommunication Engineering, University of Rome "La Sapienza", Rome, Italy \\ ${ }^{2}$ Chief Technical Office, Motorola Solutions Inc., Fort Lauderdale, FL 33322, USA \\ ${ }^{3}$ The Antenna Company Nederland B.V., Netherlands \\ ${ }^{4}$ European Space Agency, ESA-ESTEC, Keplerlaan, Noordwijk ZH, Netherlands
}

Correspondence should be addressed to Renato Cicchetti; cicchetti@die.uniromal.it

Received 11 March 2015; Accepted 11 March 2015

Copyright (c) 2015 Renato Cicchetti et al. This is an open access article distributed under the Creative Commons Attribution License, which permits unrestricted use, distribution, and reproduction in any medium, provided the original work is properly cited.

\section{Introduction}

With the advent of high data rate $3 \mathrm{G}$ and $4 \mathrm{G}$ wireless communication systems and the app-based use paradigm, wireless connectivity through multiple air interfaces has become a common requirement in the RF architecture of mobile communication devices. Modern wireless handsets frequently incorporate three or more antennas to enable cellular voice and data, Wi-Fi, and GPS connectivity, across multiple bands. Multiple antenna systems are frequently designed to implement diversity or spatial multiplexing schemes, as in the case of WCDMA and LTE, to increase resiliency and capacity of wireless links and to operate multiple voice/data links simultaneously. Concurrently, ultrawideband (UWB) systems used in short range communications, remote sensing, and through-the-wall radar imaging have introduced a new paradigm in the antenna design where the mitigation of pulse distortion is of the essence, thus requiring a shift in antenna design approach and the introduction of novel radiating systems.

This special issue is intended to reflect current $R \& D$ trends and novel approaches in the analysis and synthesis of antenna systems and associated RF front-ends for next generation mobile communication devices, applicable to various device form factors such as smartphones, tablets, laptops and wearable computers as well as for UWB communication systems and radars. A particular emphasis has been paid to the analysis and design of broadband, multiband, and reconfigurable antennas for wireless and UWB applications, as well as to the identification of special materials and integration techniques with the host platform. Important efforts have been devoted to the characterization of the radio channel as well as to the most innovative near-field-farfield transformation techniques employed to determine the radiation properties of the antennas employed in the modern wireless communication systems.

The special issue is composed of 19 contributions that can be divided into the following 9 clusters.

\section{Contributions to Broad- and Multibanding Techniques}

In "Dual-Feed Small-Size Penta-Band PIFA for LTE/WWAN Mobile Handset Applications" by M. Liu and B. Zhao, the authors present a dual-feed small-size penta-band PIFA for LTE/WWAN mobile handset applications. The proposed antenna is composed of a simple U-shaped patch with dualshorting pins connected to the ground plane. The antenna is excited at one arm of the U-shaped radiating patch to cover the higher band of GSM1800/1900/UMTS2100/LTE2300/ 2500, while it is excited at the other arm to realize the GSM900.

In "A Butterfly-Shaped Wideband Microstrip Patch Antenna for Wireless Communication" by L. Sun et al., the authors present a butterfly-shaped patch antenna for 
wideband wireless communications and radio-frequency identification (RFID) systems. The antenna, having a bandwidth of about $40 \%$, can serve simultaneously most of the modern wireless communication standards.

In "A Multiband Printed Log-Periodic Dipole Array for Wireless Communications" by G. A. Casula and P. Maxia, the authors present a compact multiband printed Log-periodic dipole array antenna for wireless communications. The proposed antenna can be used for wireless communications both in the $\mathrm{S}(2.4-3 \mathrm{GHz})$ and in the $\mathrm{C}(5.2-5.8 \mathrm{GHz})$ frequency bands, with very good input matching and a satisfactory endfire radiation pattern. In addition, it presents an excellent outof-band rejection, without the use of stop-band filters, thus avoiding interference out of its operating frequency band.

In "Bandwidth Enhancement Technique of the Meandered Monopole Antenna" by C.-J. Wang and D.-H. Hsieh, the authors present a small dual-band monopole antenna with coplanar waveguide feeding structure. The antenna is composed of a meandered monopole, an extended conductor tail, and an asymmetrical ground plane which confer to the radiating structure the ability to cover the GSM900, the DCS, the IMT-2000, the UMTS, the WLAN, the LTE 2300, and the LTE 2500, wireless communication standards.

In "Novel Cross-Type Network for Wide-Tuning-Range Reconfigurable Multiband Antennas" by C.-S. Lee et al., the authors presents a cross-type network design with a novel reconfigurable functionality to realize a tunable multiband antenna. It is shown that by attaching a reconfigurable network at the feeding port of a broadband antenna, the multi-input impedance adjustment enables the production of multimatching operating bands. So, the proposed novel cross-type network can be used successfully in tunable dualband functions to decrease the processing time and the analytical complexity of tunable-frequency devices.

In "A Compact Wideband Dual-Polarized Antenna with Harmonic Suppression Using Nonuniform Defected Ground Structure" by L. Damaj et al., the authors present an interesting dual-polarized wideband bowtie CPW-fed antenna having wideband harmonic suppression obtained by means of three nonuniform cascaded bowtie Defected Ground Structure (DGS) unit cells. The antenna has an omnidirectional dipole-type radiation pattern over the operating frequency band $2.7-5.9 \mathrm{GHz}$. These characteristics allow the proposed structure to cover the UWB (lower band in Europe) and IEEE 802.16 (WiMAX) frequency bands.

In "Design of Compact Trapezoidal Bow-Tie Chipless RFID Tag" by L. Xu and K. Huang, the authors present a novel compact design of a low-cost fully printable slot-loaded bowtie chipless RFID tag. The tag, used as an ultrawideband structure, consists of two trapezoidal metallic patches loaded with multiple slot resonators. Due to high data capacity and low cost, and since it needs only one conductive layer, it can be directly printed on personal ID, credit cards, papers, and textile fabrics.

In "A DR Loaded Substrate Integrated Waveguide Antenna for $60 \mathrm{GHz}$ High Speed Wireless Communication Systems" by N. Ashraf et al., the authors present an array of dielectric resonator antennas, excited by a compact feeding line formed by a substrate integrated waveguide, suitable to operate at $60 \mathrm{GHz}$. In particular, an impedance bandwidth of $10.70 \%$ with a gain up to $11.20 \mathrm{dBi}$ is found for an array composed of four resonator antennas.

\section{Contributions to Antennas for UWB Applications}

In "Two-Step Beveled UWB Printed Monopole Antenna with Band Notch" by Y. Xiao et al., the authors present a compact printed monopole antenna for ultra-wideband applications. Symmetrical slots, integrated along the microstrip line are employed to realize a frequency stop band, so to avoid electromagnetic interference with the WLAN communication systems, while those integrated on the ground plane are adopted to improve the antenna radiation patterns.

In "A CPW-Fed Dual-Band-Notched Antenna with Sharp Skirt Selectivity for UWB Applications" by D. Dong et al., the authors present a coplanar waveguide-fed dual-bandnotched antenna with sharp skirt selectivity for ultrawideband applications. The antenna, composed of a radiant patch with a C-shaped slot and a C-shaped stub integrated on the back surface of the substrate, presents dual-band-notched characteristics which can reject the interference between the IEEE $802.11 \mathrm{a}$ bands $(5.15-5.35 \mathrm{GHz}$ and $5.725-5.825 \mathrm{GHz})$ and the UWB systems.

\section{Contributions to Wearable/Flexible Antennas}

In "Design of a Circularly Polarized Galileo E6-Band Textile Antenna by Dedicated Multiobjective Constrained Pareto Optimization" by A. Dierck et al., the authors present a strategy, incorporating a multiobjective constrained Pareto optimization, to the design of a wearable Galileo E6-band antenna with optimal return loss and wide-band axial ratio characteristics. The realized antenna prototypes show that the proposed optimization strategy is able to take into account the variations of the antenna substrate materials.

In "Novel Multiband Metal-Rimmed Antenna for Wearable Applications" by B. Liu et al., the authors present a novel multiband antenna with an unbroken metal rim for wearable applications. The proposed antenna is composed of a radiating metal rim and a novel feeding structure which allows obtaining a compact antenna suitable to cover the frequency band between 1500 and $2350 \mathrm{MHz}$, ensuring at the same time SAR values lower than that indicated by the specific standard concerning the protection of the human being by the exposure to electromagnetic fields.

\section{Contributions to Substrates, Special Materials, and Fabrication Techniques}

In "Beam Scanning Properties of a Ferrite Loaded Microstrip Patch Antenna" by S. S. I. Mitu and F. Sultan, the authors present an axially magnetized ferrite loaded microstrip patch antenna with tunable beam scanning properties. In particular, ferrite rods are placed in the radiation region of the antenna to perturb the $E$-field phase distribution resulting in 
beam scan. The realized prototype shows a beam scan ranging from $28^{\circ}$ up to $-26^{\circ}$.

In "Design Variations on Planar Differential Antenna with Potential for Multiple, Wide, and Narrow Band Coverage" by D. Pepe et al., the authors show how by modifying the topology of an antenna it is possible to obtain a behavior presenting wide, narrow, or multiple bands. To this end, three practical antenna implementations, based on variations of one general planar differential antenna topology, originally proposed for ultrawideband applications, are presented and discussed in detail.

\section{Contributions to Reconfigurable and Multiband Antenna Technology}

In "Design of a Smart Antenna for Mobile Ad Hoc Network Applications" by M. Di Filippo et al., the authors present a smart antenna capable of producing multiple, highgain, electronically steered independent beams, made of a cylindrical array of metal patches suitable for mobile ad hoc networks. These networks are particularly important in several environments where the fixed infrastructure is not available, not trusted, too expensive, or unreliable.

\section{Contributions to MIMO Antenna Systems and Channel Modeling for Wi-Fi and LTE}

In "Polarized Uniform Linear Array System: Beam Radiation Pattern, Beamforming Diversity Order, and Channel Capacity" by X. Su and K. Chang, the authors derive the channel capacity of polarized uniform linear array (PULA) systems using the beamforming technique in a realistic scattering environment. The numerical results show that, for PULA systems, the channel capacity, which is boosted by the beamforming diversity, can be determined using the channel gain, the beam radiation pattern, and the beamforming diversity order (BDO), where the BDO is dependent on the antenna characteristics and array configurations.

\section{Contributions to Wireless Systems for Remote Control of Vital Parameters}

In "An EM Modeling for Rescue System Design of Buried People" by A. De Leo et al., the authors present an analytical model developed to get some a priori design characteristics of a rescue system, based on a R.F. detector, useful to identify the breathing activities of buried persons in case of occurrence of natural disasters such as earthquake, landslides, or avalanches. To validate the model, a system prototype was built using laboratory equipment, and some experiments were carried out in a realist scenario to confirm the performance offered in the detection of respiratory activities by means of a R.F system.

\section{Contribution to Antenna Performance Measurement Techniques}

In "Near-Field to Far-Field Transformation Techniques with Spiral Scannings: A Comprehensive Review" by R. Cicchetti et al., the authors present an overview of the most innovative spiral scanning near-field-far-field transformation techniques useful to derive the radiation patterns of the antennas commonly employed in the modern wireless communication systems. In particular, a unified theory of the spiral scannings for quasi-spherical and nonspherical antennas is described, and an optimal sampling interpolation expansion useful to evaluate the probe response on a quite arbitrary rotational surface from a nonredundant number of its samples, collected along a proper spiral wrapping it, is presented. Due to the intrinsic characteristics of the technique, a remarkable reduction of the measurement time is achieved.

\section{Contributions to Evaluation of the RF Exposure to Wireless Communication Devices and Infrastructures}

In "Culture Medium Geometry: The Dominant Factor Affecting In Vitro RF Exposure Dosimetry" by A. Paffi et al., the authors analyze the distribution of the electromagnetic field and SAR in test tubes and Petri dishes used for the exposure of cells and/or biological samples to the RF energy. In particular, they show that the RF energy distribution within the samples is entirely determined by exposure vessel geometry, orientation to the incident RF flux, frequency, and dielectric properties of the medium. The results reported in the paper are particularly useful for those researchers who are called to carry out experimental measurements on biological samples exposed to electromagnetic fields.

\section{Acknowledgments}

The editors would like to express their gratitude to the authors and the anonymous reviewers for their contributions to this special issue. As a final word to our readers, after compiling this set of papers, we believe that it gives a good and current overview of the selected topics. We hope you will enjoy reading this year's special issue as much as we have enjoyed putting it together.

Renato Cicchetti Antonio Faraone Diego Caratelli Massimiliano Simeoni 

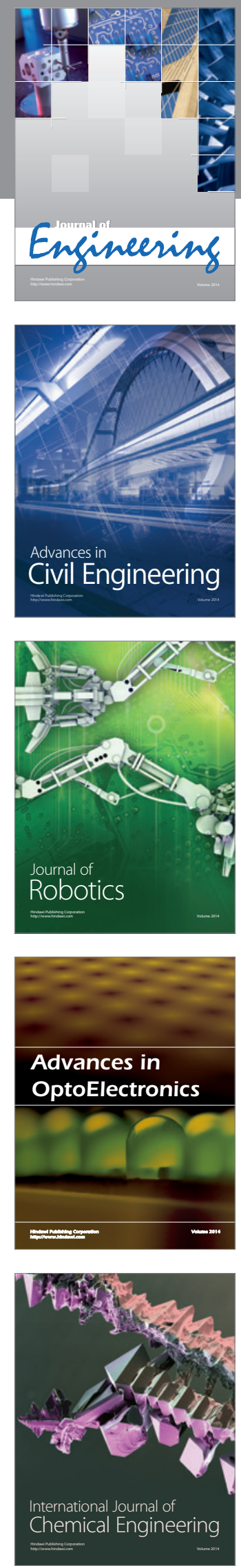

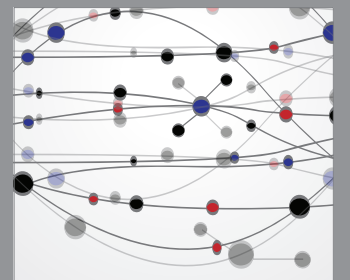

The Scientific World Journal
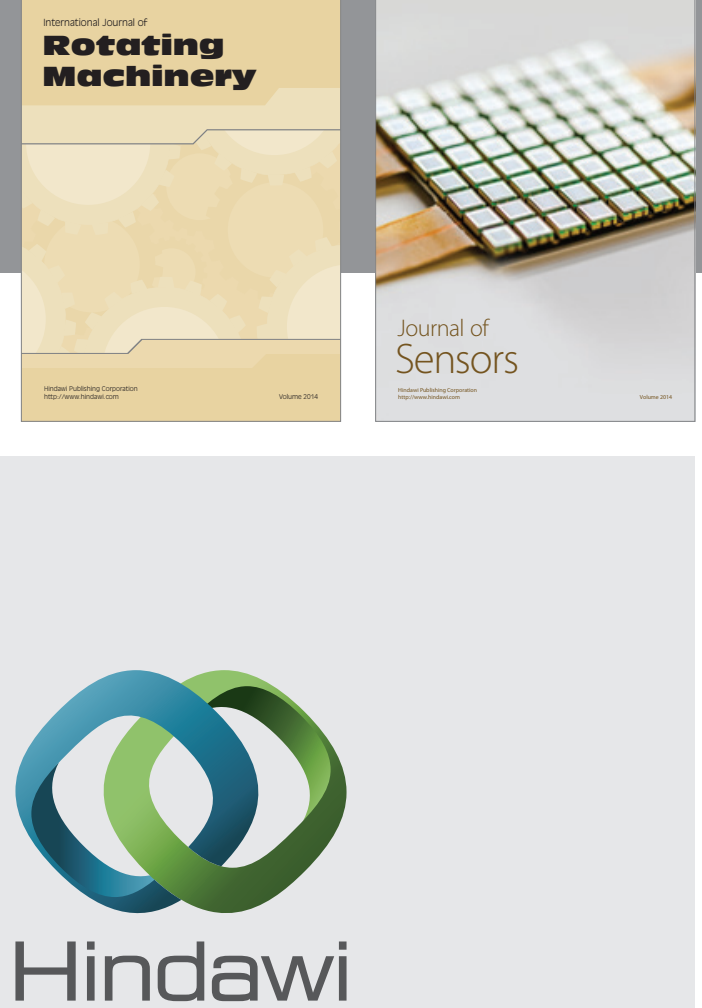

Submit your manuscripts at http://www.hindawi.com
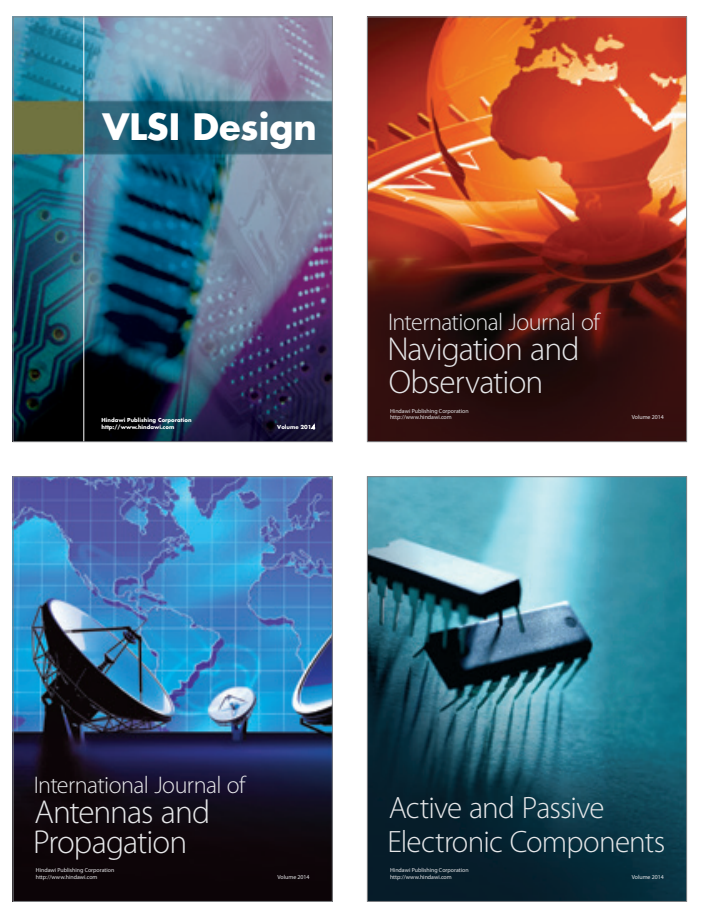
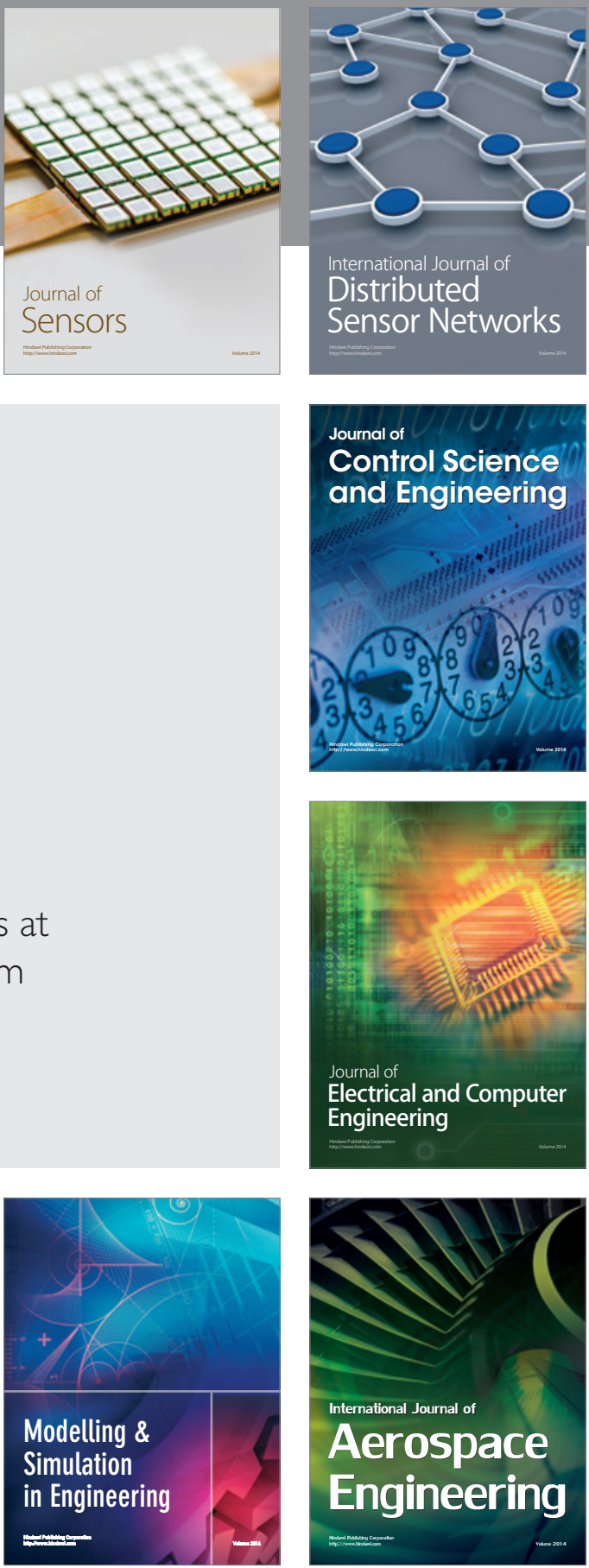

Journal of

Control Science

and Engineering
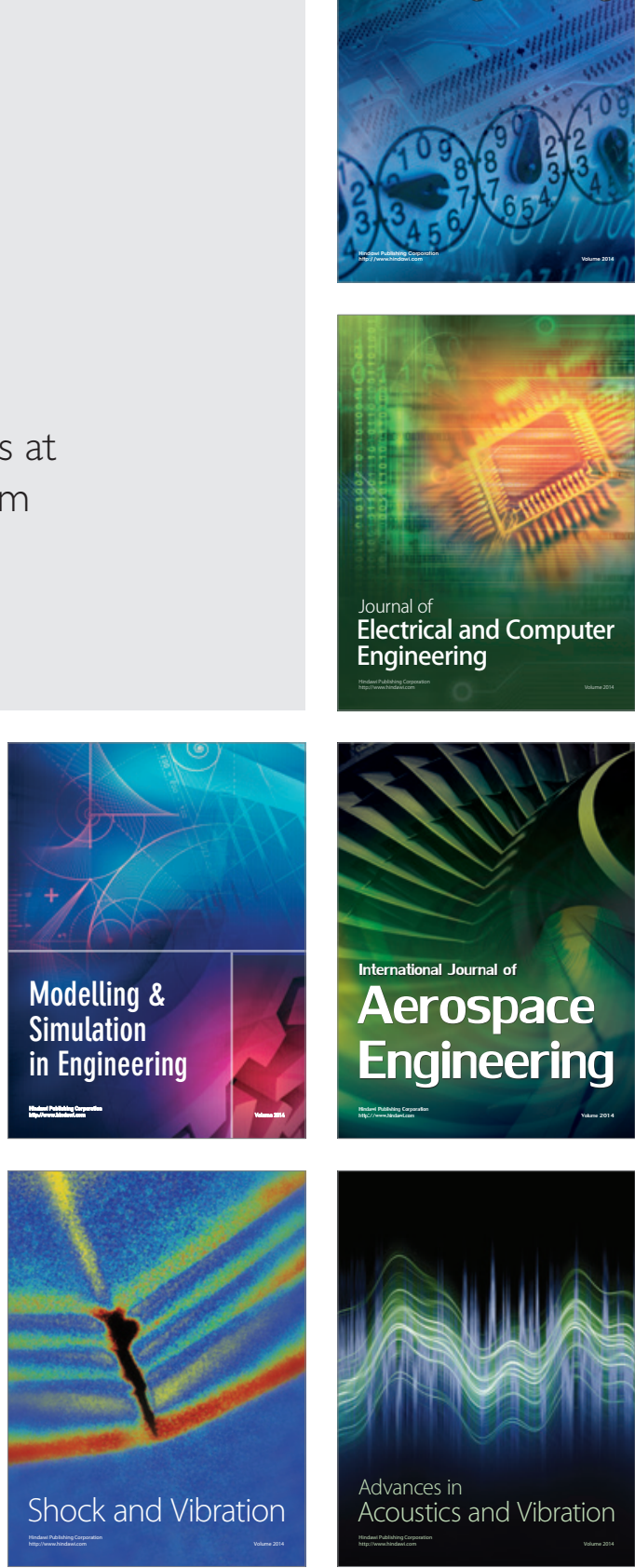